\title{
Dietary intake of vitamin D during adolescence and risk of adult onset systemic lupus erythematosus and rheumatoid arthritis
}

\author{
Linda T Hiraki ${ }^{2 *}$, Karen H Costenbader ${ }^{1}$, Kassandra L Munger ${ }^{2}$, Elizabeth W Karlson ${ }^{1}$ \\ From 2011 Pediatric Rheumatology Symposium sponsored by the American College of Rheumatology \\ Miami, FL, USA. 2-5 June 2011
}

\section{Purpose}

Early life exposures have been implicated in the etiology of rheumatoid arthritis (RA) and systemic lupus erythematosus (SLE). Vitamin D has immunomodulatory effects with implications as an etiologic and therapeutic factor in several autoimmune diseases including SLE and RA. There are no studies on vitamin D intake during adolescence and the risk of adult onset SLE and RA.

\section{Purpose}

We examined the relationship between reported vitamin $D$ intake during adolescence and incidence of adult onset RA and SLE in two prospective cohort studies, the Nurses' Health Study (NHS) and Nurses' Health Study II (NHSII).

\section{Methods}

Previously validated food frequency questionnaires (FFQ) on high school diet completed by 75,458 NHS participants in 1986 and 45,948 NHSII participants in 1998 (maximum recall of 47 and 35 years respectively), were used to calculate nutrient intakes during adolescence. Incident RA and SLE cases were confirmed medical record review. Coxproportional hazards regression relative risks (RR) and 95\% CI were calculated for quintiles of adolescent dietary vitamin D intake and incident RA, incident SLE. Calorie adjusted and multivariate adjusted analyses were completed, with adjustment for demographic, dietary and sunexposure related factors. Random effects models were used to obtain a single combined estimate of association across the two cohorts.

\section{Results}

Incident RA was confirmed in 652 NHS and 126 NHSII participants (total 778), and incident SLE was confirmed in 122 NHS and 52 NHSII participants (total 174) over a mean followup of 329 and 207 months for NHS and NHSII respectively. Calorie-adjusted and multivariateadjusted models did not show significant associations between adolescent vitamin D intake and risk of adult onset RA and SLE when comparing the 5th quintile of vitamin D intake to the 1st quintile (Table 1).

Table 1 Estimated hazard ratios and $95 \% \mathrm{Cl}$ of associated between adolescent vitamin $\mathrm{D}$ intake and incident RA and SLE

\begin{tabular}{|c|c|c|c|}
\hline & $\mathrm{NHS}$ & NHSII & Pooled \\
\hline Number of RA cases & 652 & 126 & 778 \\
\hline HR $(95 \%$ Cl) Energy adjusted Total Vitamin D & $1.03(0.8,1.32)$ & $1.06(0.62,1.82)$ & $1.03(0.82,1.3)$ \\
\hline HR (95\% Cl) Multivariate adjusted Total Vitamin D & $0.87(0.6,1.26)$ & $0.96(0.51,1.81)$ & $0.87(0.51,1.81)$ \\
\hline Number of SLE cases & 122 & 522 & 174 \\
\hline HR $(95 \%$ Cl) Energy adjusted Total Vitamin D & $1.09(0.62,1.95)$ & $1.08(0.47,2.45)$ & $1.03(0.65,1.63)$ \\
\hline HR (95\% Cl) Multivariate adjusted Total Vitamin D & $1.16(0.52,2.56)$ & $0.69(0.25,1.88)$ & $0.87(0.5,1.46)$ \\
\hline
\end{tabular}

${ }^{2}$ Harvard School of Public Health, Boston, MA, USA

Full list of author information is available at the end of the article

(C) 2012 Hiraki et al; licensee BioMed Central Ltd. This is an Open Access article distributed under the terms of the Creative Commons Attribution License (http://creativecommons.org/licenses/by/2.0), which permits unrestricted use, distribution, and reproduction in any medium, provided the original work is properly cited. 


\section{Conclusion}

We did not find associations between reported dietary intake of vitamin D during adolescence and risk of RA or SLE in adulthood. This may be due to inaccurate reporting of adolescent diet, focus on a short time period of exposure and unavailable serum vitamin D levels during this exposure period of interest. Small numbers of incident cases also reduced our power to detect an association if one existed.

\section{Disclosure}

Linda T. Hiraki: None; Karen H. Costenbader: None; Kassandra L. Munger: None; Elizabeth W. Karlson: None.

\section{Author details}

'Brigham and Women's Hospital, Harvard Medical School, Boston, MA, USA.

${ }^{2}$ Harvard School of Public Health, Boston, MA, USA.

Published: 13 July 2012

- Convenient online submission

- Thorough peer review

- No space constraints or color figure charges

- Immediate publication on acceptance

- Inclusion in PubMed, CAS, Scopus and Google Scholar

- Research which is freely available for redistribution 